\title{
Small signal stability region of power systems with DFIG in injection space
}

\author{
Chao QIN, Yixin YU ( $)$
}



\begin{abstract}
The modal analysis method is utilized to study the influence of doubly-fed induction generator (DFIG) on electromechanical oscillations. On this basis, the small signal stability region (SSSR) of power systems with DFIG in injection space is evaluated and the corresponding relationship between SSSR boundary and electromechanical oscillations is analyzed. The effects of the locations of DFIG on SSSR are considered. It is found that the boundary of SSSR consists of several smooth surfaces, which can be approximated with hyper-planes in engineering application. With the integration of DFIG, SSSR becomes smaller, thus indicating the deterioration of the small signal stability of the system. The 11-bus system with four generators is used to illustrate the proposed method.
\end{abstract}

Keywords Doubly-fed induction generator (DFIG), Modal analysis, Electromechanical oscillation, Small signal stability region (SSSR)

\section{Introduction}

In recent years, with the significant increase of wind power penetration in power systems, the influence of the doubly-fed induction generator (DFIG) [1] on small signal stability of power systems have attracted more and more attention. In [2], an analysis on small signal stability of a power system with DFIG was performed considering the influence of rotor speeds and stator resistances. The model

Received: 2 August 2012/Accepted: 10 January 2013/Published online: 12 September 2013

(C) The Author(s) 2013. This article is published with open access at Springerlink.com

C. QIN, Y. YU, Key Laboratory of Smart Grid, Tianjin

University, Tianjin 300072, China

$(\bowtie)$ e-mail: yixinyu@tju.edu.cn of DFIG for small signal stability analysis was presented in [3] and the particle swarm optimization (PSO) method was adopted to optimize the controller parameters. Reference [4] described different modeling methods of DFIG and provided relevant analysis results of small signal stability. Reference [5] investigated the impacts of different operation modes of DFIG on small signal stability of power systems. The influences of the increasing penetration of DFIG on inter-area oscillation in a small typical power system were illustrated in [6]. Reference [7] took the 11-bus system with four generators as an example and developed a control scheme for the DFIG to damp interarea oscillations. Reference [8] showed that the integration of DFIG improved the system response to small disturbances, but DFIG could absorb a large amount of reactive power after large disturbances, which affects the stability of power systems. An optimal model for controller parameters of DFIG was presented in [9] and an optimization algorithm was used to determine parameters of the exciter and pitch angle controllers of DFIG. Most researches adopt the eigenvalue analysis method to study the small signal stability of DFIG, and some of the conclusions were validated through time domain simulation. Giving the frequency and damp ratio information of oscillation modes as well as attenuation speed information of non-oscillation modes, the eigen-value analysis method reveals the mechanism of small signal stability. However, as in the scope of "point-wise" method, it can only analyze small signal stability of the power system under specified operating conditions, such as load level, power generation dispatching, etc. Once the operating conditions change, the results have to be recalculated. In recent years, the security region method [10] in power injection space is developed and applied to security and stability analysis as well as online monitoring of power systems. It overcomes the defects of "point-wise" methods and makes real-time 
security monitoring, assessment, control, and power system pricing more scientific and efficient. Besides, problems like the impact of wind power integration on power system security region, and whether the security region method can be applied to the security and stability analysis as well as power system monitoring with wind power deserve further study.

This paper investigates the influence of DFIG on electromechanical oscillations. On this basis, the small signal stability region (SSSR) of the power system with DFIG is calculated, and the relationship between SSSR boundary and electromechanical modes and the influence of DFIG on SSSR are analyzed. It is suggested to approximate the boundary of SSSR with hyper-planes in engineering application. The 11-bus system with four generators is used to illustrate the proposed method in the paper.

\section{DFIG model}

The stator side of DFIG is directly linked to the grid while the rotor side is connected to the grid through a double-pulse width modulation (PWM) inverter. The active and reactive powers of DFIG can be controlled by $d$ $q$ components of rotor current through the machine-side inverter, and the grid-side inverter is used to control the DC voltage and reactive power exchange between the rotor side of DFIG and the grid. Figure 1 shows the schematic diagram of a DFIG system. The DFIG model is shown in [11].

\section{SSSR of power systems}

Small signal stability enables a power system to remain synchronism on small and instantaneous disturbances [12]. Therefore, studies on small signal stability of a power system focus on the conditions close to the system operating equilibrium. Usually, power systems are modeled as a set of nonlinear differential algebraic equations (DAE):

$\left\{\begin{array}{l}\dot{x}=\boldsymbol{f}(\boldsymbol{x}, \boldsymbol{y}, \boldsymbol{p}) \\ \mathbf{0}=\boldsymbol{g}(\boldsymbol{x}, \boldsymbol{y}, \boldsymbol{p})\end{array}\right.$

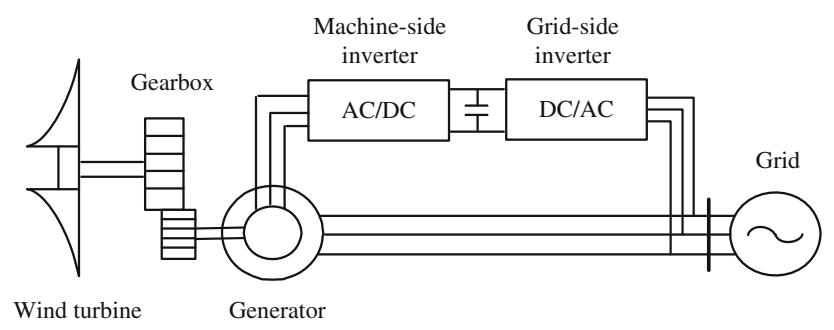

Fig. 1 Schematic diagram of a DFIG system where $\boldsymbol{x} \in \mathbf{R}^{n}$ is the state variable vector of the power system; $\boldsymbol{y} \in \mathbf{R}^{m}$ is the algebra variable vector of the power system; $\boldsymbol{p} \in \mathbf{R}^{p}$ is the decision variable vector or parameter variable vector, such as power injections, control parameters, etc.; $\boldsymbol{f}(\boldsymbol{x}, \boldsymbol{y}, \boldsymbol{p})$ is the right-hand side of the differential equation, which describes the dynamics of power systems; and $\boldsymbol{g}(\boldsymbol{x}, \boldsymbol{y}, \boldsymbol{p})$ is the algebraic equation corresponding to power flow function.

At the equilibrium point $\left(\boldsymbol{x}_{0}, \boldsymbol{y}_{0}\right)$, the DAE of a power system can be linearized as

$$
\left\{\begin{aligned}
\Delta \dot{x} & =\frac{\partial f}{\partial x} \Delta x+\frac{\partial f}{\partial y} \Delta y \\
\mathbf{0} & =\frac{\partial g}{\partial x} \Delta x+\frac{\partial g}{\partial y} \Delta y
\end{aligned}\right.
$$

Supposing that matrix $\partial g / \partial y$ is nonsingular, (2) can be expressed as

$\Delta \dot{\boldsymbol{x}}=\boldsymbol{J}(\boldsymbol{p}) \Delta \boldsymbol{x}$

where $\boldsymbol{J}(\boldsymbol{p})=\frac{\partial \boldsymbol{f}}{\partial \boldsymbol{x}}-\frac{\partial \boldsymbol{f}}{\partial \boldsymbol{y}}\left(\frac{\partial \boldsymbol{g}}{\partial \boldsymbol{y}}\right)^{-1} \frac{\partial \boldsymbol{g}}{\partial \boldsymbol{x}}$. According to eigen-value theory, the system operation state is stable if all eigenvalues of $\boldsymbol{J}(\boldsymbol{p})$ possess negative real parts.

SSSR of a power system is the set of steady operation points which can maintain small signal stability. Usually, SSSR is defined in parameter space or power injection space. In this paper, SSSR of configuration $i$ is defined in power injection space as

$$
\begin{aligned}
\Omega_{\mathrm{SSSR}}(\boldsymbol{i})= & \{\boldsymbol{p} \mid \text { all eigenvalues of } J(\boldsymbol{p}) \text { possess } \\
& \text { negative real parts, } \left.\boldsymbol{p} \in o^{2 n} \subset R^{2 n}\right\}
\end{aligned}
$$

where $\boldsymbol{p}, o^{2 n}$ and $R^{2 n}$ are complex power injection vector of the power system, feasible power injection space of the power system considering the output limits of generators and real space of $2 n$ dimensions, respectively; and $n$ is the number of power injection nodes in configuration $i$. $\Omega_{\mathrm{SSSR}}(i)$ is determined by the system configuration $i$.

Early studies of SSSR mostly focused on the solvability of power flow equation to judge small signal stability of a power system [13, 14]. Therefore, the boundary of SSSR was linked with non-oscillation instability. With the extensive application of automatic regulatory systems and FACTS, power systems are faced with oscillation instability. Therefore, the dynamic features of these equipments need to be considered. Taxonomy theory in [15] serves as a powerful tool for small signal stability study in parameter space and state space. The influences of SVC, HVDC and network dynamics on SSSR were studied in detail in [16, 17]. It was found that the saddle node bifurcation (SNB) and Hopf bifurcation (HB) are very close to each other when some nonlinear components were taken into account in [18] and that is why there are rarely reports discussing about oscillation instability of voltage in real power 


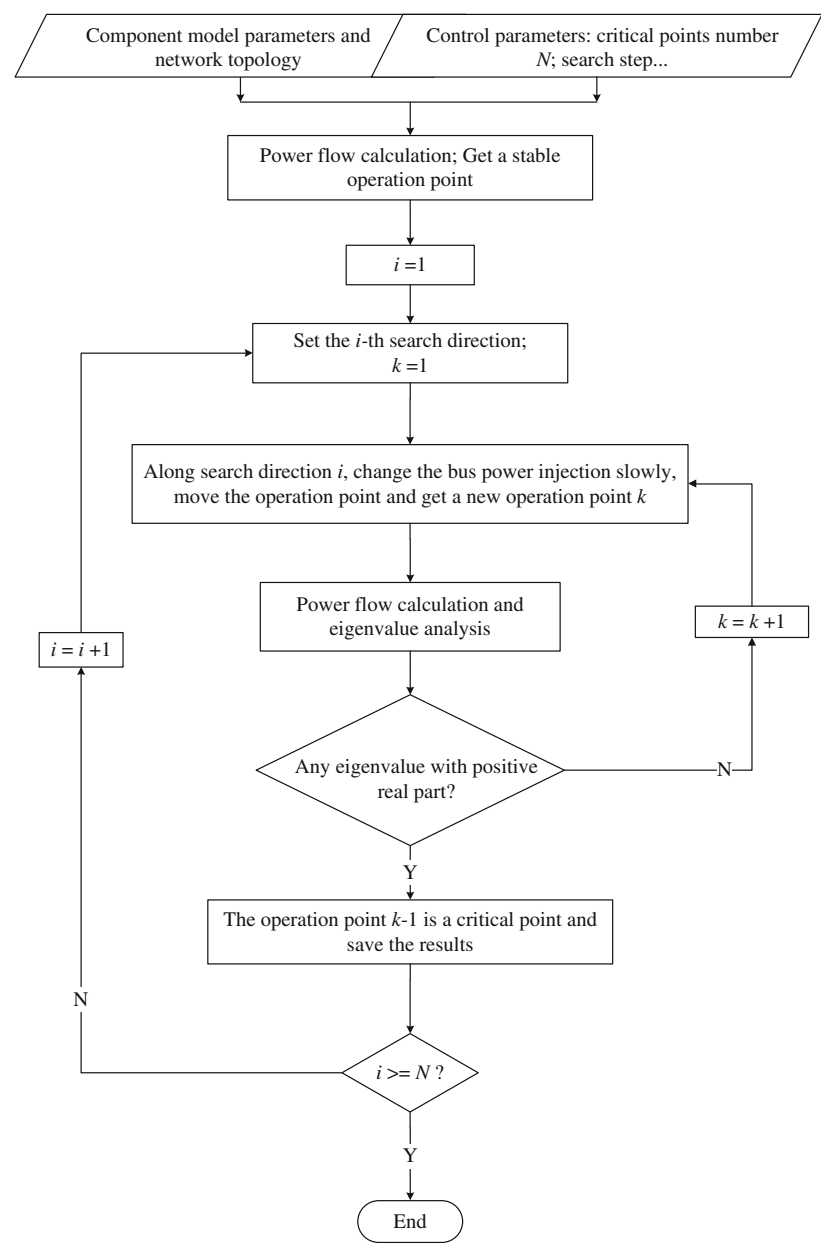

Fig. 2 Flow Chart of SSSR calculation method

system. At present, it is agreed that the boundary of SSSR mainly corresponds to three types of local bifurcation points: SNB, HB and singularity induced bifurcation (SIB).

Since the topological properties of SSSR boundary of power systems with DFIG are not clear, "point-wise" based method is adopted to evaluate the boundary of SSSR in this paper. The calculation method is shown in Fig. 2.

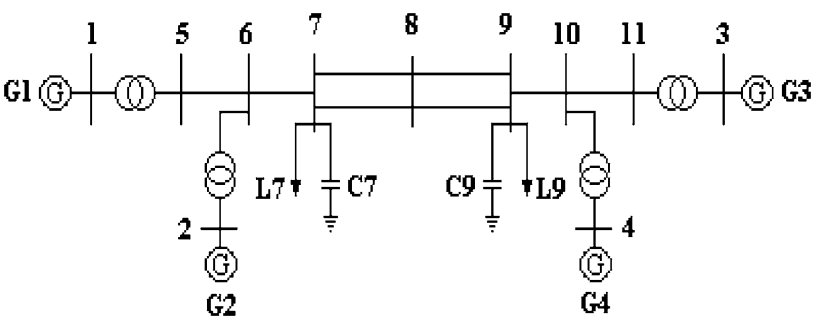

Fig. 3 Configuration of 11-bus system with four generators

\section{Case studies}

\subsection{1-bus system with four generators}

The 11-bus system with four generators is used as an illustrative example [19] and a power system analysis toolbox (PSAT) based on MATLAB is used to perform small signal stability analysis. The configuration of the 11-bus system is shown in Fig. 3. As a typical two-area system, it contains four generators and two load buses. The area of generators G1 and G2 is the side for sending power and the area of generators G3 and G4 is the side for receiving power. The detailed parameters of the system model are presented in [12].

In order to investigate the impact of doubly-fed wind farms on small signal stability of a power system, one equivalent DFIG is integrated into the system replacing part of the capacity of synchronous generators and the following three scenarios are considered.

Scenario a There is no DFIG in the system

Scenario b DFIG is connected to bus 2 through a transformer (side for sending power)

Scenario c DFIG is connected to bus 3 through a transformer (side for receiving power)

In Scenario b and Scenario c, the active power of the synchronous generator in parallel with DFIG is adjusted to keep the system operation point unchanged.

Table 1 Results of electromechanical oscillations

\begin{tabular}{|c|c|c|c|c|c|c|c|}
\hline \multicolumn{2}{|c|}{$\begin{array}{l}\text { Electromechanical oscillation } \\
\text { modes }\end{array}$} & \multicolumn{2}{|l|}{ No DFIG } & \multicolumn{2}{|c|}{ DFIG connected to bus $2(k=0.3)$} & \multicolumn{2}{|c|}{ DFIG connected to bus $3(k=0.3)$} \\
\hline No. & Involved units & Damping ratio & Frequency $(\mathrm{Hz})$ & Damping ratio & Frequency $(\mathrm{Hz})$ & Damping ratio & Frequency $(\mathrm{Hz})$ \\
\hline 1 & G1/G2 & 0.0814 & 1.0541 & 0.1107 & 1.0270 & 0.0817 & 1.0539 \\
\hline 2 & G3/G4 & 0.0796 & 1.0898 & 0.0795 & 1.0899 & 0.0963 & 1.0737 \\
\hline 3 & $\mathrm{G} 1 / \mathrm{G} 2 / \mathrm{G} 3 / \mathrm{G} 4$ & 0.0134 & 0.5345 & 0.0178 & 0.5517 & 0.0248 & 0.5963 \\
\hline 4 & $\mathrm{G} 1 / \mathrm{G} 2 / \mathrm{G} 3 / \mathrm{G} 4$ & - & - & 0.1417 & 0.3247 & -0.0951 & 0.1312 \\
\hline
\end{tabular}

Note: '-' denotes that there is no such oscillation mode 


\subsection{Modal analysis}

To study the influences of DFIG on electromechanical oscillation modes, this paper performs modal analysis on different scenarios described in Sect. 4.1. The information about electromechanical modes is shown in Table 1 , where $k$ is the proportion of DFIG output in the power injection of the connected bus.

As shown in Table 1, before the integration of DFIG, there are three electromechanical oscillation modes in the system, two of which are local oscillation modes (No. 1 and No. 2) and the other is an inter-area oscillation mode (No. 3). Moreover, the integration of DFIG introduces a new inter-area electromechanical oscillation mode (No. 4). The original inter-area oscillation mode (No. 3) is referred to as inherent global electromechanical mode and the interarea oscillation mode (No. 4) introduced by DFIG is referred to as introduction global electromechanical mode. Specially, there is an unstable electromechanical oscillation mode in Scenario c.

The damping ratio of electromechanical oscillation modes is shown in Figs. 4 and 5, respectively.

Modal analysis results illustrate that:
1) The integration of DFIG improves the damping ratio of local electromechanical oscillation modes of access region. With the increase of wind power penetration, the damping ratio also increases. It has little effect on the local electromechanical oscillation modes of the remote region.

2) Effects of DFIG on the inherent global electromechanical mode depend on the location of DFIG: when DFIG is connected to the receiving power side, the damping ratio of the mode increases while the integration of DFIG to sending power side has little effect on the mode.

3) Effects of DFIG on the introduction global electromechanical mode are as follows: when DFIG is connected to the sending power side, the damping ratio decreases with an increase of DFIG penetration, and an unstable oscillation mode appears when DFIG is connected to the receiving power side.

\subsection{Small signal stability region (SSSR)}

For the transmission system, it can be assumed that the reactive power at each node keeps locally balanced,

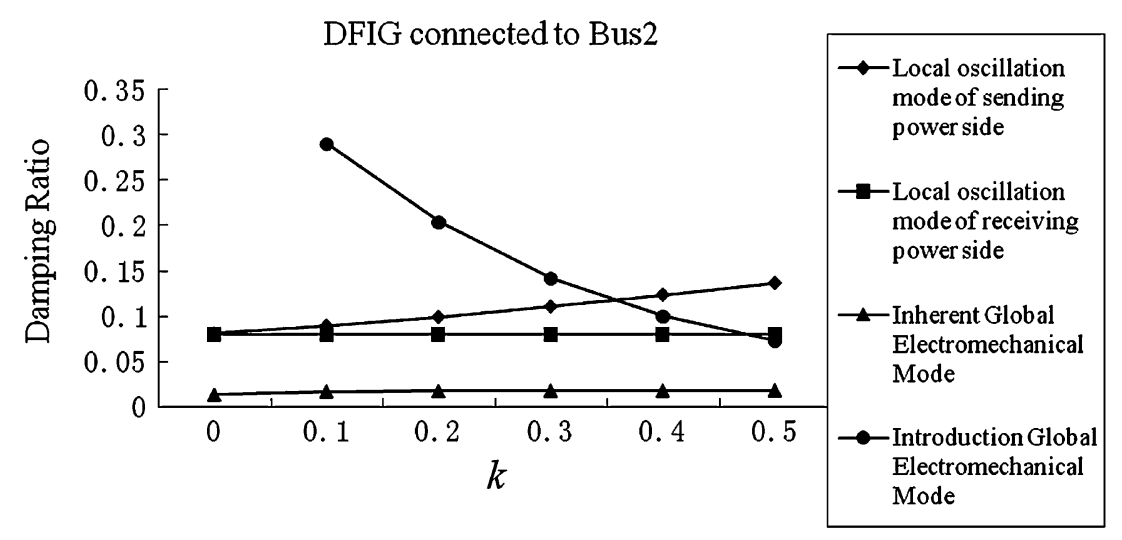

Fig. 4 Damping ratio of electromechanical oscillations (Scenario b)

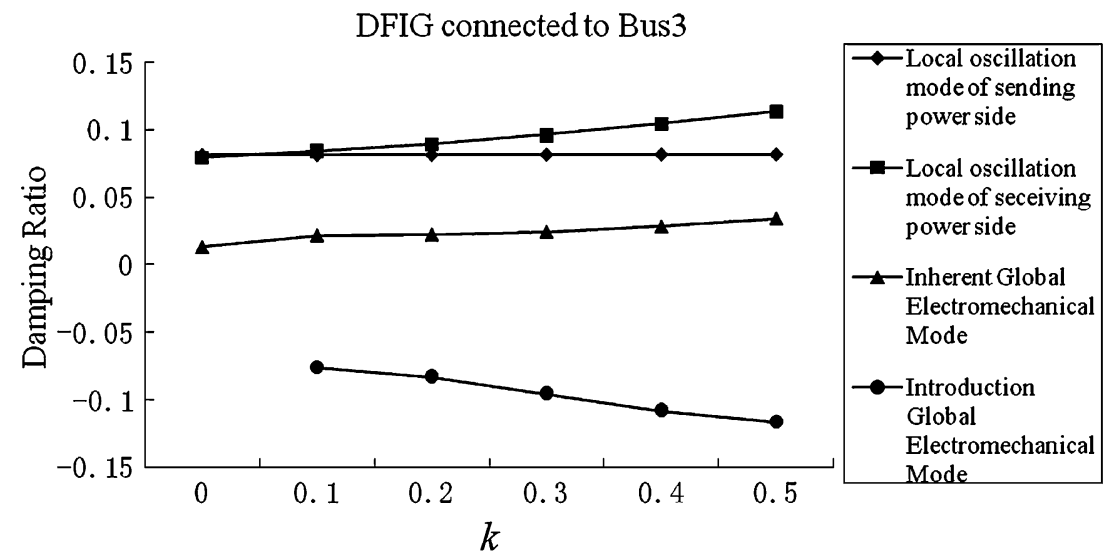

Fig. 5 Damping ratio of electromechanical oscillations (Scenario c) 


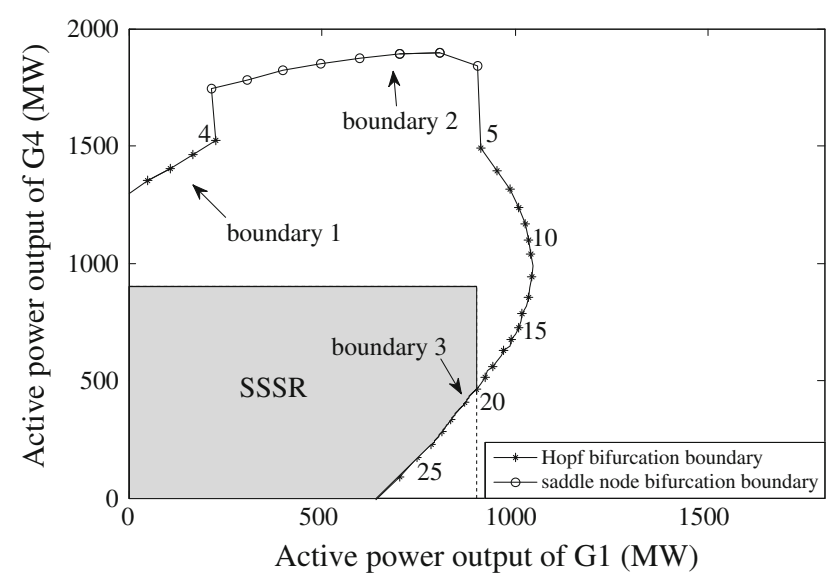

Fig. 6 SSSR boundary of scenario a



Fig. 7 Dominant oscillation modes of SSSR boundary (Scenario a)

therefore, the reactive power injections of all buses are constant. SSSR in this paper is defined in active power injection space, neglecting the power loss of the grid. The dimension of the SSSR is 5. To make better display of the boundaries of SSSR, we select the active power output of generators $\mathrm{G} 1$ and $\mathrm{G} 4$ as the variables of the power injection space and keep the others constant. In fact, the region computed in this section is a 2-dimensional crosssection of SSSR. For the system shown in Fig. 3, generator G1 is on the sending power side and G4 is on the receiving power side. Therefore, the selected 2-dimensional crosssection is a typical section.

\section{1) Scenario a}

SSSR of scenario a is shown in Fig. 6. Without considering the active power output limits, SSSR boundary consists of several smooth sections, where boundaries 1

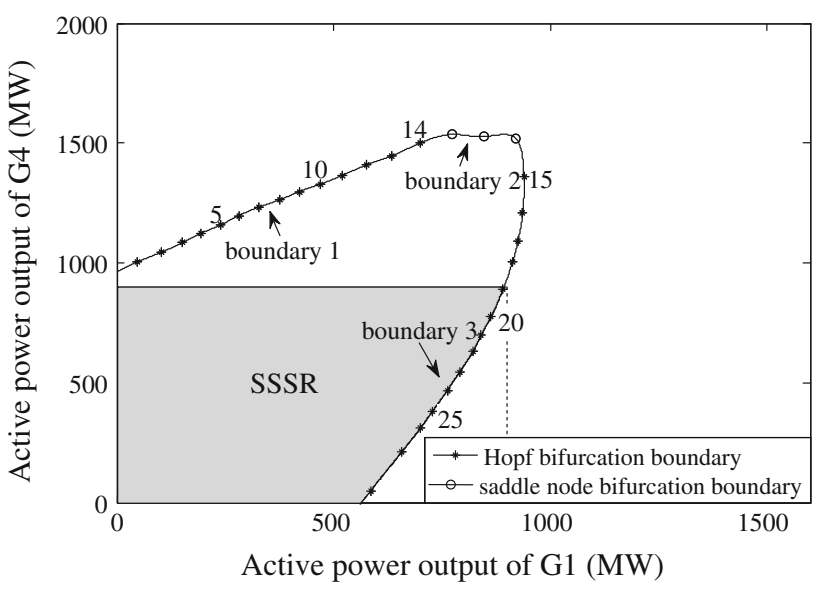

Fig. 8 SSSR boundary of Scenario b



Fig. 9 Dominant oscillation modes of SSSR boundary (Scenario b)

and 3 correspond to $\mathrm{HB}$, and boundary 2 corresponds to SNB. There are sudden changes at joints of boundaries 1 and 2 and joints of boundaries 2 and 3, for the output of excitation system of generator G4 reaches its limit. Dominant oscillation modes of boundaries 1 and 3 are shown in Fig. 7, respectively. SSSR boundary corresponds to the global electromechanical oscillation mode. Considering generator active power output limits, SSSR (shadow area) boundary consists of part of boundary 3 and the generator output limits.

2) Scenario $b$

SSSR of Scenario $\mathrm{b}(k=0.3)$ is shown in Fig. 8 .

Without considering the active power output limits of generators, SSSR boundary consists of three smooth curves and two axes. The three smooth curves correspond to two oscillation modes and a non-oscillation mode. The dominant 


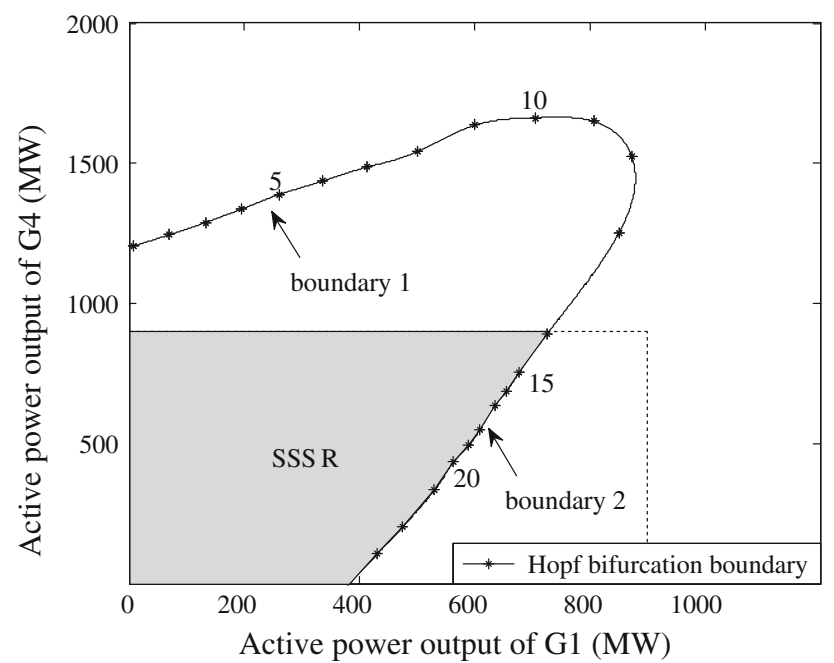

Fig. 10 SSSR boundary of scenario c

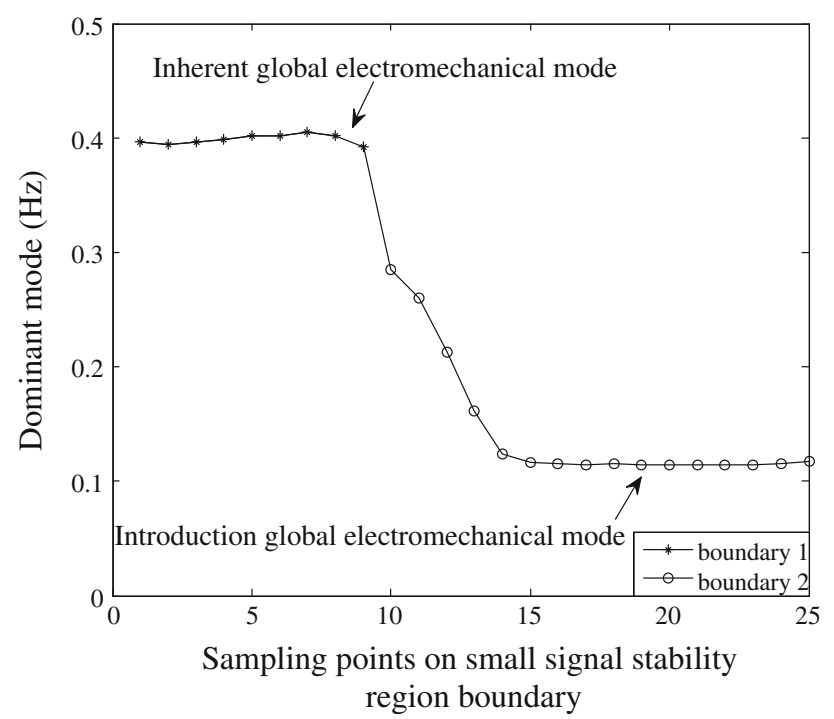

Fig. 11 Dominant oscillation modes of SSSR boundary (Scenario c)

modes of boundaries 1 and 3 are shown in Fig. 9. Boundary 1 is linked to the inherent global electromechanical mode while boundary 3 corresponds to introduction global electromechanical mode. Considering the generator active power output limits, the boundary of SSSR (shadow area) consists of part of boundary 3 and the generator output limits.

\section{3) Scenario c}

As shown in Figs. 10 and 11, SSSR of Scenario c $(k=0.3)$ consists of two smooth boundary parts and two axes. And boundary 1 corresponds to the inherent global electromechanical mode and boundary 2 is linked to introduction global electromechanical mode. Taking into account the active power output limits of generators, SSSR (shadow area)

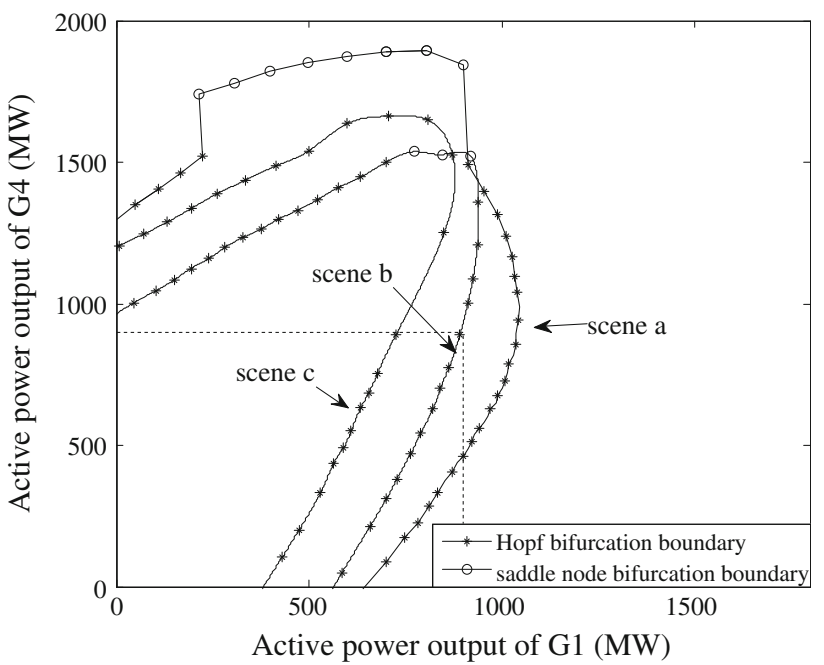

Fig. 12 Comparison of SSSR boundaries

Table 2 Fitting results for SSSR boundary

\begin{tabular}{lllllll}
\hline Scenario & $\alpha_{1}$ & $\alpha_{2}$ & $\alpha_{3}$ & $\alpha_{4}$ & $\alpha_{5}$ & $\begin{array}{l}\text { Max fitting } \\
\text { error }(\%)\end{array}$ \\
\hline $\mathrm{a}$ & 0.1655 & 0.1535 & 0.0199 & 0.1167 & 0.041 & 1.15 \\
$\mathrm{~b}$ & 0.2198 & 0.2227 & 0.0063 & 0.1519 & 0.0247 & 1.07 \\
$\mathrm{c}$ & 0.2159 & 0.2133 & 0.0330 & 0.1528 & 0.0394 & 1.96 \\
\hline
\end{tabular}

boundary consists of part of boundary 2 and the generator output limits. Therefore, when DFIG is connected to receiving power side, the influences of DFIG are shown through the introduction global electromechanical mode.

4) Comparison of SSSR boundary

As shown in Fig. 12, in engineering applications, SSSR becomes smaller when DFIG is connected to the system, which means that the small signal stability of the power system will deteriorate.

\section{5) Hyper-plane fitting}

Reference [20] studied the hyper-plane fitting of the boundary of security region for traditional power systems. Whether SSSR of the power system with DFIG can be fitted by hyper-planes is a matter of concern. Simulation results show that the 2-dimensional cross-sections of SSSR are all of smooth characteristics. Therefore, the following inference can be drawn: in $n$-dimensional power injection spaces, SSSR of the power system with DFIG can still be fitted by hyper-planes in engineering applications. In case of neglecting power loss of the grid, the hyper-plane fitting results in $n$-dimensional injection spaces can be expressed as $\alpha_{1} P_{1}+\alpha_{2} P_{2}+\cdots+\alpha_{n-1} P_{n-1}=1$ 
The fitting error $\left(E r r_{\mathrm{SSSR}}\right)$ is defined as

$$
\operatorname{ErrSSR}_{\mathrm{SSS}}=\frac{\left|\sum_{i=1}^{n-1} \alpha_{i} P_{i}-1\right|}{\sqrt{\sum_{i=1}^{n-1} \alpha_{i}^{2}} \cdot \sqrt{\sum_{i=1}^{n-1} P_{i}^{2}}}
$$

where $P_{i}(i=1,2, \ldots, n-1)$ is active power injection of Bus $i$ and $\alpha_{i}(i=1,2, \ldots, n-1)$ is the hyper-plane coefficient.

For the system studied in this paper, the active powers of buses 1, 2, 4, 7,9 are selected as the power injection variables and Bus 3 is the swing bus. The least squares method is used to approximate the critical points selected with reference to orthogonal table with hyper-plane [21] (There may be more than one hyper-plane, and the one with most critical points is selected as the approximation of SSSR boundary). As shown in Table 2, the max fitting error is $1.96 \%$, which satisfies the needs of engineering applications. Therefore, SSSR of the power system with DFIG can be approximated by hyperplanes in engineering applications.

\section{Conclusion}

In this paper, the impact of DFIG on electromechanical oscillation modes of power systems are studied, and a new global electromechanical oscillation mode caused by the integration of DFIG is determined. Based on detailed studies of SSSR for power systems with DFIG, some of the relationships between SSSR boundary and electromechanical oscillation modes are characterized: (1) the boundary of SSSR of power systems with DFIG consists of several smooth surfaces; (2) in engineering applications, the boundary of SSSR can be approximated by hyper-planes; (3) the integration of DFIG makes SSSR smaller, which means it has negative impact on the small signal stability of power systems; (4) the location of DFIG has certain effects on SSSR of a power system.

Acknowledgments This work is supported by National Basic Research Program of China (973 Program) (No. 2009CB219701).

Open Access This article is distributed under the terms of the Creative Commons Attribution License which permits any use, distribution, and reproduction in any medium, provided the original author(s) and the source are credited.

\section{References}

[1] Pena R, Clare JC, Asher GM (1996) Doubly fed induction generator using back-to-back PWM converters and its application to variable-speed wind-energy generation. IEE Proc Electr Power Appl 143(3):231-241

[2] Mei F, Pal BC (2007) Modal analysis of grid-connected doubly fed induction generators. IEEE Trans Energy Convers 22(3):728-736
[3] Wu F, Zhang XP, Godfrey K et al (2007) Small signal stability analysis and optimal control of a wind turbine with doubly fed induction generator. IET Gener Transm Distrib 1(5):751-760

[4] Mei F, Pal BC (2005) Modeling and small-signal analysis of a grid connected doubly-fed induction generator. In: Proceedings of IEEE PES General Meeting, San Francisco

[5] Mishra Y, Mishra S, Li F et al (2009) Small-signal stability analysis of a DFIG-based wind power system under different modes of operation. IEEE Trans Energy Convers 24(4):972-982

[6] Tsourakisa G, Nomikosb BM, Vournasa CD (2009) Effect of wind parks with doubly fed asynchronous generators on smallsignal stability. Electr Power Syst Res 79(1):190-200

[7] Miao Z, Fan L, Osborn D et al (2009) Control of DFIG-based wind generation to improve interarea oscillation damping. IEEE Trans Energy Convers 24(2):415-422

[8] Elkington K, Knazkins V, Ghandhari M (2008) On the stability of power systems containing doubly fed induction generatorbased generation. Electr Power Syst Res 78(9):1744-1784

[9] Lin J, Li G, Sun Y et al (2009) Small-signal analysis and control system parameter optimization for DFIG wind turbines. Autom Electr Power Syst 33(5):86-90 (in Chinese)

[10] Yu YX (2008) Review of study on methodology of security regions of power system. J Tianjin Univ 41(6):635-646 (in Chinese)

[11] Slootweg JG (2003) Wind power: modeling and impact on power system dynamics. Doctorial Dissertation. Delft University of Technology, Delft, The Netherlands

[12] Kundur P (1994) Power system stability and control. McGrawHill, New York, pp 699-825

[13] Jarjis J, Galiana FD (1981) Quantitative analysis of steady state stability in power networks. IEEE Trans Power Appar Syst 100(1):318-326

[14] Kwatny HG, Fischl RF, Nwankpa CO (1995) Local bifurcation in power systems: theory, computation, and application. Proc IEEE 83(11):1456-1483

[15] Venkatasubramanian V, Schattler H, Zaborszky J (1995) Dynamics of large constrained nonlinear systems-a taxonomy theory. Proc IEEE 83(11): 1530-1561

[16] Arabi S, Rogers GJ, Wong DY et al (1991) Small signal stability program analysis of SVC and HVDC in AC power systems. IEEE Trans Power Syst 6(3):1147-1153

[17] Parniani M, Iravani MR (1995) Computer analysis of smallsignal stability of power systems including network dynamics. IEE Gener Transm Distrib 142(6):613-617

[18] Jia HJ, Yu YX, Wang CS (2001) The chaotic and bifurcation phenomena considering power system excitation limit and PSS. Autom Electr Power Syst 25(1):11-14 (in Chinese)

[19] Milano F (2005) An open source power system analysis toolbox. IEEE Trans Power Syst 20(3):1199-1206

[20] Sun Q, Yu YX (2008) Hyper-plane approximation of boundary of small signal stability region and its application. J Tianjin Univ 41(6):647-652 (in Chinese)

[21] Yu YX, Luan WP (1990) Practical dynamic security regions of power systems. Proc CSEE 10(supp):22-28 (in Chinese)

\section{Author Biographies}

Chao QIN is currently a Ph.D. candidate in Electrical Engineering and Automation School of Tianjin University. His main research interests include power system security and stability, wind power.

Yixin YU is a professor of Tianjin University. He became an Academician of Chinese Academy of Engineering in 2005. His research interests include smart grid, power system security and stability, power system planning and deregulation. 